\title{
AWMF-Leitlinie zur radiologischen Diagnostik im Kopf-Hals-Bereich
}

Die S1-Leitlinie „Radiologische Diagnostik im Kopf-Hals-Bereich“ ist eine gute Hilfestellung sowohl für radiologisch tätiges Personal und Zahnärzte/Zahnärztinnen als auch für die zuweisenden Kollegen/Kolleginnen. Sie soll bei der Entscheidung über den Einsatz und die Reihenfolge bildgebender Verfahren unterstützen und ist eine gute Ergänzung zur Orientierungshilfe der Strahlenschutzkommission.

Für die wichtigsten Erkrankungstypen, die für die verschiedenen Regionen des KopfHals-Bereichs abgehandelt werden, sind in Übersichtsform Empfehlungen für die effektive und zweckdienliche Anwendung der verschiedenen Verfahren zusammengefasst.

Die AWMF-Leitlinie finden Sie unter: www.vmtb.de. 